\title{
Pediatric presentation of a large intrathoracic mass
}

\author{
Norma L. Cooney • Derek R. Cooney
}

Received: 16 April 2009/Accepted: 2 May 2009/Published online: 30 May 2009

(C) Springer-Verlag London Ltd 2009

A 4-year-old girl presented with a 3-month history of shortness of breath with exertion and chest pain for 1 day. There were no other associated symptoms. The patient had no significant past medical history. The patient had presented to an outside institution 2 months previously and was diagnosed with reactive airway disease. On physical exam, vital signs showed mild tachycardia, tachypnea, and normal oxygen saturations. The patient was anxious. Her voice was non-hoarse. The patient's chest was slightly prominent and there were slightly distant heart sounds. The remainder of the physical exam was normal. Labs were unremarkable. Chest X-ray showed a markedly widened mediastinum and cardiomegaly (Fig. 1). Computed tomography of the chest confirmed a large calcified anterior mediastinal mass with mass effect on the distal trachea and a moderate pericardial effusion.

N. L. Cooney $(\bowtie)$

Department of Emergency Medicine,

SUNY Upstate Medical University,

750 East Adams Street,

Syracuse, NY 13210, USA

e-mail: cooneyn@upstate.edu

D. R. Cooney

Department of Emergency Medicine,

EMS \& Disaster Medicine,

SUNY Upstate Medical University,

EMSTAT Center/550 East Genesee,

Syracuse, NY 13202, USA

e-mail: cooneyd@upstate.edu

URL: http://www.upstate.edu/emergency/residency/fellowship/

emsdm.php
The presence of calcified density components led to the diagnosis of teratoma. The initial diagnosis of reactive airway disease in this 4-year-old with no history of atopy or previous airway disease should lead to further investigation. A chest X-ray may have allowed for the correct diagnosis early in the presentation. Mediastinal tumors are classified as posterior, middle, superior, and anterior. Tumors specifically of the anterior region consist of teratomas, dermoid cysts, and hemangiomas. Anterior and superior mediastinal tumors require admission. Concern and special attention should be paid to airway management. If intubation is required, advanced airway techniques may need to be utilized. Bronchoscopic intubation may be necessary especially if there is compromise of the airway. "Adminis-

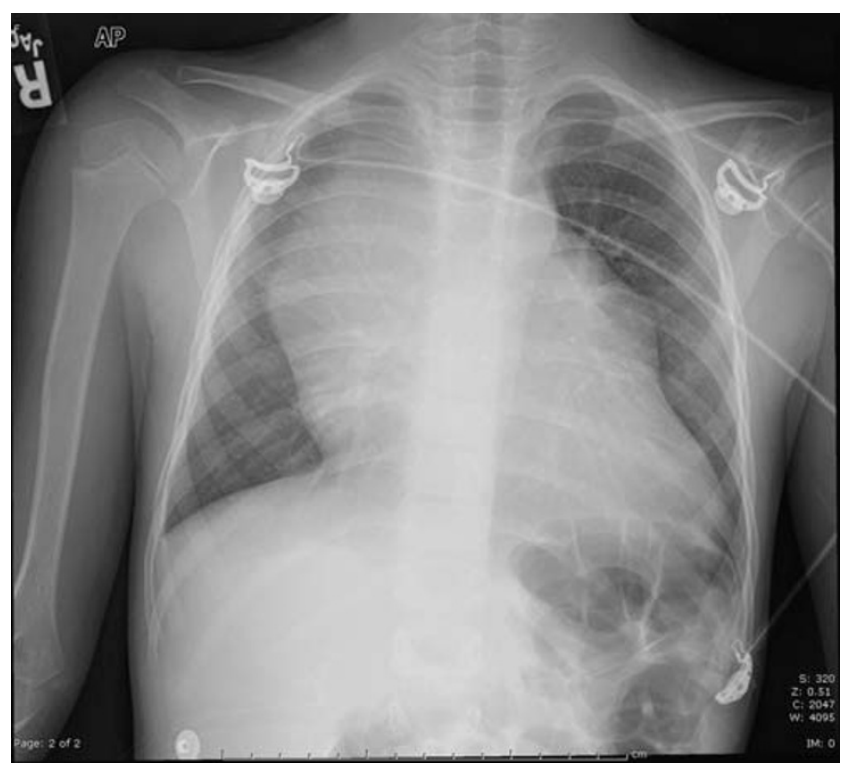

Fig. 1 Chest X-ray 
tering anaesthesia to a child with an anterior mediastinal mass may lead to respiratory or circulatory collapse, even in those without symptoms [1]." Evaluations by hematology/ oncology and pediatric surgery are warranted.

\section{References}

1. Hammer GB (2004) Anaesthetic management for the child with a mediastinal mass. Paediatr Anaesth 14(1):95-97 